\title{
Reduction of micro-object's adhesion using chemical functionalisation
}

\author{
Jérôme Dejeu, Patrick Rougeot, Michaël Gauthier, Wilfrid Boireau \\ FEMTO-ST Institute, UMR CNRS 6174 - UFC / ENSMM / UTBM, \\ 24 rue Alain Savary, 25000 Besançon, France \\ Phone: +33 (0)3 814028 10, Fax: +33 (0)3814028 09, \\ E-mail: michael.gauthier@femto-st.fr
}

\begin{abstract}
The adhesion and interaction properties of functionalised surfaces (substrate or cantilever) were investigated by means of atomic force microscope (AFM) related force measurements. The surfaces were functionalised with a polyelectrolyte: Poly(Allylamine Hydrochloride) (PAH), or with silanes: 3 (ethoxydimethylsilyl) propyl amine (APTES) or (3 aminopropyl) triethoxysilane (APDMES). Measurements of forces acting between a bare glass sphere (functionalised or not) and a functionalised surface indicated repulsive or attractive forces, depending on functionnalisation and medium (wet or dry). Adhesion forces (pull-off) can be observed in dry medium while in wet medium, this phenomenon can be cancelled. Now, the pull-off forces is an important problem in the automation of micro-object manipulations. The cancellation of this force by chemical functionnalisation is thus a promising way to improve micro-assembly in the future.
\end{abstract}

\section{Introduction}

Manufactured products are becoming always smaller and are integrating more and more functionalities in small volumes. Several application fields are concerned by the Micro-Electro-Mechanical-Systems (MEMS) such as the telephony, bio-engineering, telecommunications. The assembly of these microproducts is a great challenge because of the microscopic sizes of the components. In fact, the major difficulties of micro-assembly come from the particularity of the micro-object's behaviours which depend on surface forces $[1,2,3]$. The manipulation of a micro-object requires 
to handle, position, and release it without disturbances of the surface forces such as electrostatic forces, van der Waals forces or capillary forces. The release is the more critical phase which is usually hindered by adhesion.

Several methods have been proposed in the last ten years to improve micromanipulations $[4,5]$. The first approach consists in using non-contact manipulations like laser trapping [6] or dielectrophoresis [7]. These manipulation methods are not disturbed by adhesion but the blocking force stays low which is a major drawback when applied in microassembly. The second approach deals with contact manipulation where the adhesion is reduced or directly used for manipulation. The reduction of the adhesion can be achieved for example by adding roughness on the end-effectors $[8,9]$. Adhesion can be directly used to perform manipulations. In this case, one-fingered gripper is sufficient to handle objects, but the release stays difficult [10]. The release requires in fact new methods such as the inertial release [11] or dielectrophoresis release [12]. In contact handling, the blocking force is usually high. The current microhandling methods are able to improve micromanipulations but the object's behaviour is always disturbed by adhesion and the reliability is still low $[9,13]$.

We propose a new way in contact handling which consists in chemically controlling the surface forces between the object and the gripper. The major objective is to reduce the adhesion force or to create a repulsive force to guarantee a reliable release. This paper shows that the tested functionnalisations are able to significantly reduce adhesion. As adhesion is the major disturbance in micro-handling, this fact directly shows the potentiality of using functionalised microgripper to assemble microparts. This paper based on interaction force measurements consequently shows the potentiality of this approach.

Now, the surface properties of a material can be controlled by surface functionnalisation. The surface functionnalisation of the objects or of the grippers can be obtained by different methods. The two more important methods are the physisorption of polyelectrolyte (polyelectrolyte with positive or negative charges) [14] or the grafting of molecules on the surface (covalent bound between the substrate and the molecules) [15, 16, 17] in order to form a self-assemble monolayers. This phenomenon is mainly due to two processes depending of the instrinsic atomic compositions of the substrates: Oxydized substrates are useful for silane functionalisation whereas gold substrates are only reactive with sulfhydrils compounds. The difference in reactivity could be a real advantage in our approach because the using of silane (or thiol) molecules will allow a vectorization of the chemical processes. This could be a highly versatile tool box to give specific properties to a precise part of the micro-objects especially in the real world of micro-assembling.

The first method induces electrostatic interaction between the substrate and the polyelectrolyte. There is no bounding between the substrate and the adsorbed molecules. The electrostatic interaction can be modified during the adsorption or after by the $\mathrm{pH}$ and the ionic strength of the bulk, and by the humidity in dry medium. The polyelec- 
trolyte used can be strong (totally ionised whatever the $\mathrm{pH}$ or the ionic strength) or weak (ionisation changed with the $\mathrm{pH}$ or the ionic strength). The polyelectrolytes adsorption takes place in aqueous solution. So two substrates can be functionalised with complementary charges (positive for one and negative for the other one) or identical charges in order to generate electrostatic interaction respectively attractive or repulsive forces. Usually, several polyelectrolyte layers are necessary in order to increase the attractions of the microstructures. The second method generates covalent bound between substrate and molecules. These molecules must contain silanol, thiol, azide, allyl or vinyl groups $[15,16]$ in an extremity. These molecules have to be used in organic solvent as toluene, acetone, methanol, ethanol, ... The silanol create a bound Si-O-Si with the silica substrate [15] while allyl or vinyl generates Si-O-C (or Si-C) bound [18] and the azide groups produce Si-N bound [19].

Consequently, we are proposing to improve micromanipulations by using functionalised objects and gripper to control contact interaction. This article focuses on the feasibility of this approach based on the measurement of attractive and repulsive force in function of the medium. The chemical surface functionnalization is presented in the following section. Experiments and control of the interactive force using the medium are showed in the section 3.

\section{Experimental procedures}

\subsection{Materials and chemicals}

The polyelectrolyte, Poly(Allylamine Hydrochloride) (PAH), with an average molecular weight of $70 \mathrm{kDa}$ and the silanes: 3 (ethoxydimethylsilyl) propyl amine (APTES) and (3 aminopropyl) triethoxysilane (APDMES), figure 1, ethanol and sodium chloride $(\mathrm{NaCl})$, were purchased from Sigma Aldrich. The three chemical compounds (PAH, APTES, APDMES) used to surface functionnality are an amine function $\mathrm{NH}_{2}$ which can protonated or ionised to $\mathrm{NH}_{3}^{+}$according to $\mathrm{pH}$. In acidic $\mathrm{pH}$, the anime is totally ionised, then the ionisation decreases and is null in basic pH (between pH 9 and 12). The deposits were made on SiFiT (silicon Fingers Tips) design in the Department. The Milli Q water was obtained with the Direct-Q 3 of Millipore.

\subsection{Surface functionalisations}

Before being functionalised, the wafers were cleaned by immersion in a piranha solution (2 parts $\mathrm{H}_{2} \mathrm{SO}_{4}, 1$ part $\mathrm{H}_{2} \mathrm{O}_{2}$ ) during 25 minutes at $70^{\circ} \mathrm{C}$. Then, the wafers were rinsed, stored in Milli Q water before $\mathrm{PAH}$ functionalisation or rinsed in milli Q water and in ethanol before silanisation (functionalisation by silane: APDMES, APTES). Solutions were freshly prepared by direct dissolution in milli-Q quality water and in ethanol for respectively the PAH and the 


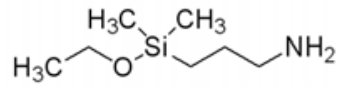

(a) APDMES

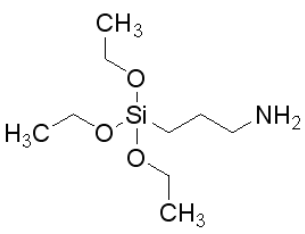

(b) APTES

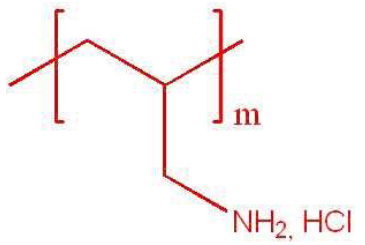

(c) $\mathrm{PAH}$

Figure 1: Molecules used for the silica functionnalisations.

silanes. The final silanes concentration was of $1 \%$. The PAH solution was a polymer concentration of $10 \mathrm{mg} / \mathrm{ml}$, and ionic strength of $0.5 \mathrm{M}$ in $\mathrm{NaCl}$, and a $\mathrm{pH}$ of 9 . The surfaces were functionalised by immersion in solutions during one night at room temperature. In the polymer solution, the PAH was just adsorbed in the surface (no covalent bound) and in the silanes solution, the silanes were gratfed on the substrate (covalent bound). The excess of ungrafted silanes was removed by ultrasonication during 2 mins in ethanol.

\section{3 functionalisation mechanisms}

\subsubsection{PAH adsorption}

The protonation of the $\mathrm{PAH}$ is partial at $\mathrm{pH} 9$ (near $40 \%$ ) [20,21]. In the bulk the $\mathrm{NH}_{2}$ functions of the $\mathrm{PAH}$ were ionised to $\mathrm{NH}_{3}^{+}$. When the substrate (silica last layer), negatively charged from $\mathrm{pH} 5$ [22], is immersed in the cationic PAH solution, the cationic polymer chains are electrostatically attracted by the negative charges covering the substrate. Thus, silica layer counter-ions, are substituted by cationic polymer chains, transported by diffusion near the substrate, and a surface ion pairing occurs between polymer chains and silica negative charges. Consequently, the mechanism of deposition is based on the substitution of the silica counter-ions by charged polyelectrolyte. This fixation is possible thanks to the electrostatic affinity between the cationic PAH and the negatively charged substrate. It is the physisorption reaction with no covalent bound.

\subsubsection{Grafted silanes}

The mechanism of self-assembled monolayers formation during silanization process is depicted in figure 2, which takes place in four steps [23, 24]. The first step is physisorption, in which the silane molecules get physisorbed at the hydrated silicon surface. In the second step, the silane head-groups arriving close to the substrate hydrolyse in the presence of the adsorbed water layer on the surface, into highly polar trihydroxysilane $\mathrm{Si}(\mathrm{OH})_{3}$ or hydroxysilane 
$\mathrm{Si}(\mathrm{OH})(\mathrm{Me})_{2}$ for respectively triethoxysilane $\mathrm{Si}(\mathrm{OEt})_{3}$ (APTES) and ethoxysilane $\mathrm{Si}(\mathrm{OEt})$ (APDMES). These polars groups $\left(\mathrm{Si}(\mathrm{OH})_{3}\right.$ or $\left.\mathrm{Si}(\mathrm{OH})\right)$ form covalent bonds with the hydroxyl groups on $\mathrm{SiO}_{2}$ surface (third step) subsequently to which condensation reaction (release of water molecules) goes on between silanol functions of neighbour molecules. Self-assembly is driven by lipophilic interactions between the linear alkane. During initial period, only a few molecules will adsorb (by steps 1-3) on the surface and the monolayer will definitely be in a disordered (or liquid) state. However, at longer times, the surface coverage eventually reaches the point where a well-ordered and compact (or crystalline) monolayer is obtained (step 4), for APTES only, by the condensation reaction between the APTES molecules.
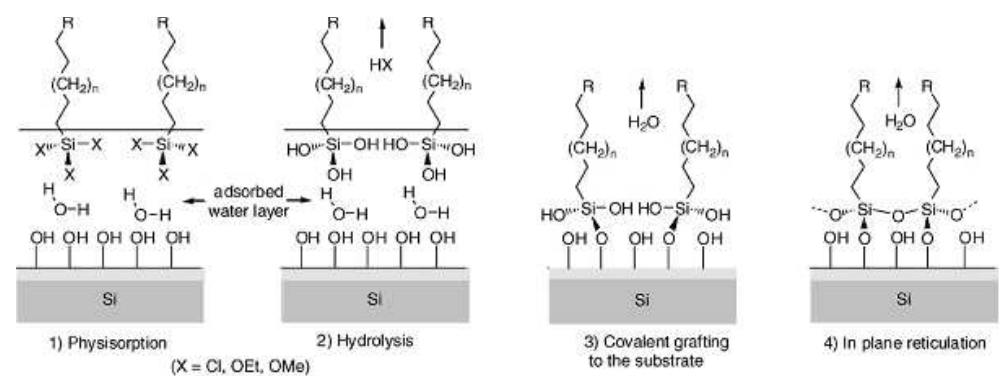

Figure 2: Steps involved in the mechanism of SAM formation on a hydrated silicon surface [23, 24].

\subsection{Force-Distance Measurement}

Force-distance measurement were performed using a stand-alone SMENA scanning probe microscope (NT-MDT) in order to characterize the functionalisation. The AFM has an optical deflection measure with a laser diode, a beam and a Sensitive PhotoDiode. The SPD is a sensor with 4 quadrants shapes giving 2 values for one deflection (normal (flexion) and one lateral (torsion) signal). The AFM cantilever, whose stiffness is $0.3 \mathrm{~N} / \mathrm{m}$, was fixed and the sample moved vertically. Each measurement was performed with a silicon rectangular cantilever (Novascan Technologies, Ames, USA) where a borosilicate sphere (borosilicate, $10 \mu \mathrm{m}$ diameter) was glued onto the apex of the tipless (Ref.:PT.BORO.SI.10, company Novascan Technologies, Ames, USA). As applicative objective of this work is to improve reliability of micro-object manipulation, interactions have been studied between a micrometric sphere (10 $\mu \mathrm{m}$ diameter) and a plane. All measurements were done at the driving speed of $200 \mathrm{~nm} / \mathrm{s}$ to avoid the influence of the hydrodynamic drag forces $[25,26]$ in liquid medium. Experiments in air were done with humidity percent near $30 \%$. For each sample, nine measures were done in different points. The repeatability of all the measure of pull-off and pull-in forces was better than $10 \%$. 


\section{Experimental results}

\section{1 functionalised surface}

We started with the study of the interaction and adhesion properties in dry and wet media. The force distance curves were measured just after functionalisation of the surface with a non fonctionnalised cantilever. The surface functionalisation modifies the interaction between the surface and the cantilever. Indeed, before functionalisation, there is a less repulsion between the two surfaces because of the negative charge on the surface due to the silanol groups, and after surface functionalisation, we measured attractive forces (near -20 nN, figure 3.b) for all the chemical functionalisation used. An example is presented in Figure 3 for the APTES gratfed silane on silica. The point 0 on the distance axis corresponds to the contact point between the cantilever and the surface.

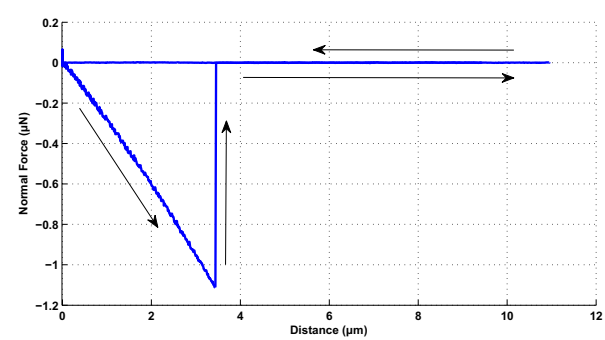

(a) Total curve

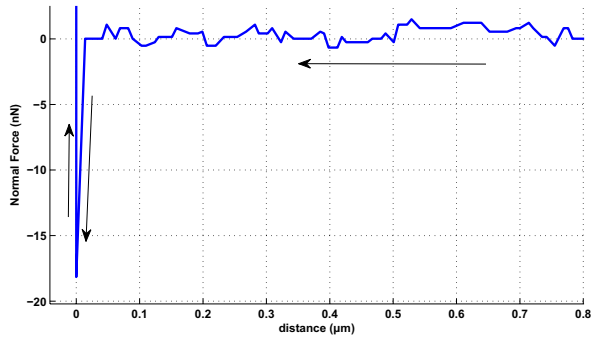

(b) near the contact point

Figure 3: Force-distance curves on the substrate functionalised APTES in dry medium obtained with a tip which spring constant is $0.3 \mathrm{~N} / \mathrm{m}$.

In Figure 3, there is no hysteresis been forces measured on approach and on retract. we clearly measured a pull-off force which represents the adhesion between the glass sphere on the tip and the functionalised substrate. For example in Figure 3.a , the pull-off force is reading $1.1 \mu \mathrm{N}$. The same experiments were done with the other chemical compounds (adsorpted or grafted molecules on the substrate) in wet and dry media. The average of the nine different measures realized on three places, adhesion and approach forces, for the two mediums, are summarized in the table 1 .

The pull-in and pull-off forces change with the functionalisation and the medium. Indeed, in dry medium, the pull-in forces are low (about ten nano Newtons) perhaps because the charge is compensated by counter ions and their mobility is very low. The adhesion, in dry medium, of the surface functionalised with APTES is more important than PAH and APDMES. This difference can be explained by the fact that the layer of APTES reaches the point 


\begin{tabular}{|c|c|c|c|c|}
\cline { 2 - 5 } \multicolumn{1}{c|}{} & \multicolumn{2}{c|}{ Dry medium } & \multicolumn{2}{c|}{ Wet medium } \\
\hline functionalisation & Pull-in $(\mathrm{nN})$ & Pull-off $(\mathrm{nN})$ & Pull-in $(\mathrm{nN})$ & Pull-off $(\mathrm{nN})$ \\
\hline APDMES & $-4.97 \pm 1$ & $-769 \pm 72$ & $-29.8 \pm 6$ & $-353 \pm 21$ \\
\hline APTES & $-13.2 \pm 1.5$ & $-1150 \pm 90$ & $-59.5 \pm 8$ & $-387 \pm 38$ \\
\hline PAH & $-11.7 \pm 1.1$ & $-720 \pm 67$ & $-24.3 \pm 6$ & $-310 \pm 27$ \\
\hline
\end{tabular}

Table 1: Influence of the surface functionalisation in dry and wet medium on the Pull-in (nN) and Pull-off (adhesion forces $)(\mu \mathrm{N})$ obtained with a tip whose spring constant is $0.3 \mathrm{~N} / \mathrm{m}$.

where a well-ordered and compact (or crystalline) monolayer is obtained (step 4, Figure 2), and by the fact that during the surface functionalisation, the silanisation reaction can be done between one silane grafted with the surface and free silane not grafted in solution.

In wet medium, the pull-in forces increased but they didn't arrive at very important values because the $\mathrm{pH}$ of the Milli Q water is near $\mathrm{pH} 5.5$ and at this $\mathrm{pH}$, the silica charges are not very important. The pull-off forces decrease compared to dry medium, because of the increase of the mobility of the counter ions. Indeed, the counter ions can more rapidly compensate the cantilever and the surface charges during the surface retract.

To conclude on this part, the micro-assembly of structures could be done in wet medium which decreases their adhesion. In order to manipulate and to assemble micro-objects, we are going to show that the adhesion can also be completely cancelled by functionalisation of both substrate and sphere on AFM cantilever.

\section{2 functionalised surface and cantilever}

The cantilever functionalisation was only done with the APTES silane and with no sonification step. The forcedistance curves were realized in the same conditions as previously, dry and wet medium, three surfaces functionalised (APTES, APDMES, PAH). In this case, there are no attraction but repulsion between all the functionalised surface and functionalised cantilever. The Figure 4 presents the example of the PAH surface in wet medium.

We observe a repulsion (positive pull-in force near $0.8 \mu \mathrm{N}$ ) and no pull-off force between the two funtionnalised surfaces, and a slight hysteresis between forces measured on approach and retract. The average values of the nine different measures realized on three places, (pull-in and pull-off forces), for the two mediums, are summarized in the table 2.

In this table 2, the pull-in forces are null in dry medium and appear clearly repulsive in wet medium. There are not important differences between the three chemical compounds on pull-in force in liquid medium. The cantilever and the surface have the same positive charges due to the anime functions which are practically completely ionised in MilliQ water ( $\mathrm{pH}$ 5.5), so in the form $\mathrm{NH}_{3}^{+}$. However, the pull-off forces with $\mathrm{PAH}$ adsorded on the subtraste 


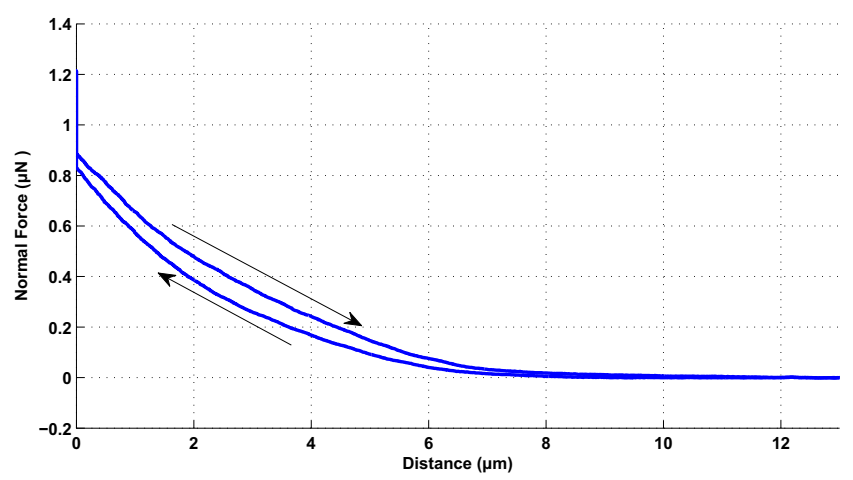

Figure 4: Force-distance curve for the PAH functionalised substrate in wet medium obtained with a tip whose spring constant is $0.3 \mathrm{~N} / \mathrm{m}$.

\begin{tabular}{|c|c|c|c|c|}
\cline { 2 - 5 } \multicolumn{1}{c|}{} & \multicolumn{2}{c|}{ Dry medium } & \multicolumn{2}{c|}{ Wet medium } \\
\hline functionalisation & Pull-in $(\mathrm{nN})$ & Pull-off $(\mathrm{nN})$ & Pull-in $(\mu \mathrm{N})$ & Pull-off $(\mathrm{nN})$ \\
\hline APDMES & 0 & $-136 \pm 35$ & $735 \pm 60$ & 0 \\
\hline APTES & 0 & $-91 \pm 23$ & $655 \pm 50$ & 0 \\
\hline PAH & 0 & $-801 \pm 58$ & $774 \pm 67$ & 0 \\
\hline
\end{tabular}

Table 2: Influence of the surface functionalisation on APTES grafted on cantilever in dry and wet medium on the Pull-in $(\mathrm{nN})$, Pull-off $(\mu \mathrm{N})$ obtained with a tip which spring constant is $0.3 \mathrm{~N} / \mathrm{m}$.

is more important than the surface with silane grafted and it is near the same value with no functionnality on the cantilever (table 1). We can explain this, by the fact that PAH is just adsorbed on the surface and not grafted. So when the cantilever is in contact with the surface, the PAH molecules adsorbed on the cantilever and on the surface move in order to create the better interaction between surface and cantilever as in the case surface was functionalised and cantilever free of molecules.

The cancellation of the pull-off force in wet medium is a great opportunity in the application field of micromanipulation. In fact, the handling of functionalised micro-objects with functionalised grippers in a liquid could be done without adhesion disturbances. Future works will focus on the application of this first result on experimental micromanipulations in order to definitively show the relevance of functionalized grippers in robotic microhandling.

\section{Conclusion}

In this paper, we have studied adhesion behaviours between functionalised surfaces and functionalised or neutral micrometric sphere. The experiments were done in wet and dry medium. The surface are functionalised by three 
chemical compounds using two different ways of functionalisation: adsorption of polyelectrolytes (PAH) and silanisation (grafted of silane molecules). We have shown that the functionalisation and the medium could highly change the adhesion properties. The micro-assembly could be facilitated by a judicious choice of the media and of the functionalisation of grippers and micro-objects. If the object is not functionalised, the manipulation in a wet media can, in fact, reduce the pull-off forces. Pertubations will be thus reduced in wet medium. Moreover, if the object is functionalised, the wet medium can be used to simply cancel the adhesion. As adhesion is the current highest pertubation phenomenon in micromanipulation, functionalisation is a promising way to improve micro-object manipulation in the future.

\section{Acknowledgement}

This work was supported by the EU under HYDROMEL contract NMP2-CT-2006-026622 : Hybrid ultra precision manufacturing process based on positional- and self-assembly for complex micro-products, and by the French National Agency (ANR) under NANOROL contract ANR-07-ROBO-0003: Nanoanalyse for micromanipulate.

\section{References}

[1] P. Lambert. Capillary Forces in Micro-assembly. Springer, 2008.

[2] Michaël Gauthier, Stephane Régnier, Patrick Rougeot, and Nicolas Chaillet. Forces analysis for micromanipulations in dry and liquid media. Journal of Micromechatronics, 3:389-413, 2006.

[3] Q. Zhou, B. Chang, and H. N. Koivo. Ambient environment effects in micro/nano handling. In Proc. of the Int. Workshop on Microfactories, pages 146-51, Shangai, China, 2004.

[4] Veikko Sariola, Quan Zhou, and Heikki N. Koivo. Hybrid microhandling: a unified view of robotic handling and self-assembly. Journal of Micro - Nano Mechatronics, 4:5-16, 2008.

[5] Pierre Lambert. A case study of surface tension gripping: the watch bearing. Journal of Micromechanical Microengineering., 16:1267-1276, 2006.

[6] Kishan Dholakia, Peter Reece, and Min Gu. Tutorial reviewoptical micromanipulation. Chem. Soc. Rev., $37: 42-55,2008$.

[7] T. P. Hunt and R. M. Westervelt. Dielectrophoresis tweezers for single cell manipulation. Biomedical Microdevices, 8:227-230, 2006. 
[8] F. Arai, D. Andou, Y. Nonoda, T. Fukuda, H. Iwata, and K. Itoigawa. Integrated microendeffector for micromanipulation. IEEE/ASME Transactions on Mechatronics, 3:17-23, 1998.

[9] David Hériban and Michaël Gauthier. Robotic micro-assembly of microparts using a piezogripper. In Proc. of the 2008 IEEE/RSJ International Conference on Intelligent Robots and Systems, pages 4042-4047, Nice, France, 2008

[10] Pierre Lambert and Stephane Régnier. Surface and contact forces models within the framework of microassembly. International Journal of Micromechatronics, 3:123-157, 2006.

[11] W. Driesen, T. Varidel, S. Régnier, and J.M. Breguet. Micromanipulation by adhesion with two collaborating mobile micro robots. Journal of Micromechanics and Microengineering, 15:259-267, 2005.

[12] M. Gauthier, E. Gibeau, and D. Hériban. Submerged robotic micromanipulation and dielectrophoretic microobject release. In Proc. of the IEEE ICARCV 2006 conference, Singapour, 2006.

[13] Mélanie Dafflon, Benoit Lorent, and Reymond Clavel. A micromanipulation setup for comparative tests of microgrippers. In International Symposium on Robotics (ISR), 2006.

[14] J.B. Schlenoff G. Decher. Sequential Assembly of Nanocomposite Materials. Wiley-VCH, 2003.

[15] Gökhan Demirel, Mustafa O. Caglayan, Bora Garipcan, and Erhan Piskin. A novel dna biosensor based on ellipsometry. Surface Science, 602:952-959, 2008.

[16] J.H. Maas, M.A. Cohen Stuart, A.B. Sieval, H. Zuilhof, and E.J.R. Sudhölterb. Preparation of polystyrene brushes by reaction of terminal vinyl groups on silicon and silica surfaces. Thin Solid Films, 426:135-139, 2003.

[17] T. Mangeat, A. Berthier, C. Elie-Caille, M. Perrin, W. Boireau, Ch. Pieralli, and B. Wacogne. Gold/silica biochips: Applications to surface plasmon resonance and fluorescence quenching. Laser Physics, 19:1-7, 2009.

[18] O. Moreau, C. Portella, F. Massicot, J.M. Herry, and A.M. Riquet. Adhesion on polyethylene glycol and quaternary ammonium salt-grafted silicon surfaces: Influence of physicochemical properties. Surface \& Coatings Technology, 201:5994-6004, 2007.

[19] Jing Wang, Dong-Jie Guo, Bing Xia, Jie Chao, and Shou-Jun Xiao. Preparation of organic monolayers with azide on porous silicon via sin bonds. Colloids and Surface A : Physicochemical and Engineering Aspects, 305:66-75, 2007.

[20] Alexander I. Petrov, Alexei A. Antipov, and Gleb B. Sukhorukov. Base-acid equilibria in polyelectrolyte systems: From weak polyelectrolytes to interpolyelectrolyte complexes and multilayered polyelectrolyte shells. Macromolecules, 36:10079-10086, 2003. 
[21] Jérôme Dejeu. Films de deux polymères auto-assemblés : chlorydrate de polyallylamine et polystyrène sulfonate de sodium; mécanisme de croissance et stabilité,. PhD thesis, University of Franche Comte, 2007.

[22] A. Foissy and J. Persello. The Surface Properties of Silicas. John Wiley \& Sons, 1998.

[23] S.R. Wasserman, Y.-T. Tao, and G.M. Whitesides. Structure and reactivity of alkylsiloxane monolayers formed by reaction of alkyltrichlorosilanes on silicon substrates. Langmuir, 5:1074-1087, 1989.

[24] N. Tillman, A. Ulman, and J.S. Schildkraut ans T.L. Penner. Incorporation of phenoxy groups in self-assembled monolayers of trichlorosilane derivatives. effects on film thickness, wettability, and molecular orientation. Journal of American Chemical Society, 110:6136-6144, 1988.

[25] O. I. Vinogradova, H. J. Butt, G. E. Yakubov, and F. Feuillebois. Dynamic effects on force measurements. 1. viscous drag on the atomic force microscope cantilever. Review of Scientific Instruments, 72:2330-2339, 2001.

[26] Olga I. Vinogradova and Gleb E. Yakubov. Dynamic effects on force measurements. 2. lubrication and the atomic force microscope. Langmuir, 19:1227-1234, 2003. 